\title{
Der Aufbau, das systematische Zentrum und die Gegenwartsrelevanz der Confessio Augustana
}

Die Tagung, für die dieser Beitrag angefragt und konzipiert ist, stand unter der Frage, ob es sinnvoll ist, das von Erwin Iserloh und anderen verfolgte Anliegen einer Anerkennung der Katholizität der Confessio Augustana durch die römischkatholische Kirche wieder aufzunehmen und weiterzuverfolgen. ${ }^{1}$,Katholizität ${ }^{\text {* }}$ soll dabei vermutlich bedeuten: die Anerkennung, dass die in der Confessio $\mathrm{Au}$ gustana vorgetragenen Lehren dem entsprechen, was nach dem Commonitorium des Vinzenz von Lerinum semper, ubique et ab omnibus creditum est. ${ }^{2}$ Damit stellt sich natürlich nicht nur die Frage, ob dies der Fall ist, sondern es stellt sich die Frage, ob dieser Anspruch, den die Väter der CA mit Sicherheit und ausdrücklich erhoben haben, ${ }^{3}$ denn in der Gegenwart von den protestantischen Kirchen so erhoben wird, die die grundsätzliche Anerkennung der Geltung der reformatorischen Bekenntnisse durch die Aufnahme in die Grundordnung ihrer Kirchen bekräftigen, diesen Geltungsanspruch aber doch mit grundsätzlichen hermeneutischen Erwägungen relativieren, in Einzelfällen ausdrücklich bestreiten und

1 In der Veranstaltungsankündigung hieß es: „Zum 450. Jubiläum im Jahr 1980 gab es - inspiriert durch die ökumenische Bewegung - eine umfangreiche und intensive Diskussion über die Frage einer Anerkennung der Katholizität der ,Confessio Augustana', eine Diskussion, die abrupt und ergebnislos endete. Nach dem gewachsenen ökumenischen Vertrauen durch das Reformationsjahr 2017 stellt sich die Frage, ob wir im Blick auf 2030 hinsichtlich einer ökumenischen Anerkennung der Katholizität der CA einen Schritt weiterkommen können. Das Symposium soll eine Diskussion eröffnen, die in Kirche, Wissenschaft und Gemeindeleben weitergeführt und vertieft werden muss.“ http://www.melanchthon.com/Veranstaltungen/2019/2019-10-10-Tagung-Confes sio.php (19.06.2021).

2 In ipsa item ecclesia catholica magnopere curandum est ut id teneatur quod ubique, quod semper, quod ab omnibus creditum est [...] Sed hoc ita demum fit, si sequamur universitatem antiquitatem consensionem. - (In der katholischen Kirche ist größte Sorgfalt darauf zu verwenden, daß das festgehalten wird, was überall, was immer, und was von allen geglaubt worden ist. [...] Das wird dann geschehen, wenn wir dem [Leitfaden von] Universalität, Alter und Übereinstimmung folgen.). Commonitorium pro catholicae fidei antiquitate et universitate adversus profanas omnium haereticorum novitates, hg.v. A. Jülicher, Tübingen ${ }^{2} 1925$ (neueste kritische Edition: CCSL 64), Bd. 2, 5-6.

3 BSLKrev 130,8-27 // BSLK 83c-d; im Folgenden zitiere ich diese und weitere lutherische Bekenntnisschriften nach der revidierten Ausgabe, hg.v. Irene Dingel, Göttingen 2014. Ich ergänze jedes Zitat aus den Bekenntnisschriften durch den Verweis auf die ,Jubiläumsausgabe‘ von 1930.

๑ OpenAccess. ( 2022 Notger Slenczka, publiziert von De Gruyter. (cc) BY-NC-ND Dieses Werk ist lizenziert unter einer Creative Commons Namensnennung - Nicht kommerziell - Keine Bearbeitung 4.0 International Lizenz. https://doi.org/10.1515/9783110683868-031 
spätestens seit dem 18. Jahrhundert die Frage nach der gegenwärtigen Verbindlichkeit historisch abständiger Texte mit offenem Ausgang diskutieren. ${ }^{4}$ Damit stellt sich die viel grundsätzlichere Frage, in welchem Sinne man für ,den christlichen Glauben' Katholizität im Sinne des Vinzenz von Lerinum in Anspruch nehmen kann: liegt diese Katholizität tatsächlich auf der Ebene gegenständlicher Lehrinhalte? Aller Inhalte beispielsweise des Apostolikums - zu schweigen von der CA, den übrigen Bekenntnisschriften oder der Lehrentscheidungen des Denzinger? Sofern die Katholizität nicht in diesen Inhalten liegt - wo dann? Weiter: was bedeutet es, die Katholizität der CA in der Gegenwart anzuerkennen? Wird damit die Entwicklung und Ausbildung von Konfessionskirchen, die einander als different und unvereinbar betrachtet haben, rückgängig gemacht? Wird damit eine Einheit konstatiert, für die die nach 1530 bzw. in der Neuzeit eingetretene Entwicklung der Lehrbildung hinsichtlich der Funktion des Papstes oder der Rolle der Mutter Gottes einerseits und die entsprechenden Entwicklungen auf protestantischer Seite bedeutungslos sind? Soll das historische Bekenntnis von 1530 gleichsam die hermeneutische Klammer werden, unter der alle weiteren Entwicklungen zu bewerten sind? Oder werden die seit 1530 vollzogenen Entwicklungen zum hermeneutischen Schlüssel des Verständnisses der CA? Hätte das nicht die Folge, dass sich der gewonnene Konsens bald wieder aufreibt?

Ich notiere diese Fragen, denen viele weitere zur Seite zu stellen wären, nur darum, um deutlich zu machen: das Projekt der Anerkennung der Katholizität der CA ist ökumenisch und hermeneutisch anspruchsvoll; es zeichnet sich ab, dass eine schlichte Ja-Nein-Antwort auf die Frage, ob die CA ,katholisch` ist, ohnehin und mit Gründen nicht möglich ist.

Mein Vortrag soll zur Klärung dieser Frage durch eine Verständigung über das systematische Zentrum der CA beitragen, also die Frage nach der ,Mitte der CA stellen und beantworten. Schon diese Teilfrage ist voraussetzungsreich: sie impliziert nämlich die Behauptung, dass die CA keine Versammlung einzelner Lehren ist, über deren ,Katholizität‘ man Artikel für Artikel entscheiden könnte. Die CA gruppiert ihre Artikel vielmehr um ein inhaltliches Zentrum, dem alle Artikel zugeordnet sind. Das bedeutet, dass es, gemessen an diesem Zentrum, mehr oder weniger zentrale Inhalte gibt, und dass alle Lehraussagen unter dem hermeneutischen Kriterium dieses Zentrums stehen. Dass diese Perspektive wieder voraussetzungsreich ist, ist klar: Es stellt sich die Frage, ob das Identifizieren eines solchen hermeneutischen Zentrums wirklich die Intention der Verfasser oder der rezipierenden Kirchen des 16. und 17. Jahrhunderts widerspiegelt,

4 Dazu Notger Slenczka, Theologie der reformatorischen Bekenntnisschriften, Leipzig 2020, hier $\S 1$ und $\S 11$. 
oder ob es sich um eine nachträgliche Sinnstiftung handelt, die nur nach dem Überschreiten nicht der zeitlichen, sondern der inhaltlichen Schwelle zum Neuprotestantismus möglich ist. Um das Identifizieren des Zentrums möglichst weitgehend von Willkürakten freizustellen, erschließe ich es über eine Analyse des Aufbaus der CA und trage ein paar Beobachtungen vor, die noch selten gemacht wurden und nicht selbstverständlich sind (2.). Fragen nach dem Aufbau von Texten sind darum hilfreich für deren Interpretation, weil sie nicht an der Frage nach der historisch (vielleicht) feststellbaren Absicht des Verfassers oder der Verfasser orientiert ist, sondern an der Instanz des Textes und seiner Struktur, die weder durch historische noch durch systematische Zugriffe ignoriert werden kann und beiden vorausliegt. Allerdings setzt die auswertende Interpretation dieses Aufbaus die Bestimmung der historischen Situation bzw. der Absicht der Abfassung voraus, die daher in 1 . soweit wie nötig nachvollzogen werden soll. Auf dieser Grundlage wird das Zentrum der CA erkennbar (3.). Ich werde dann versuchen, die Gegenwartsrelevanz dieses Zentrums zu bestimmen (4.) - ich konzentriere mich auf die ökumenische Relevanz. Daraus ergibt sich unter 5. eine Vermutung über die systematische Funktion des Genus ,Lehrbekenntnis‘. Und es ergibt sich (6.) die Einsicht, dass die katholische Kirche sehr schlecht beraten wäre, wenn sie die CA als kirchliches Bekenntnis anerkennen würde.

Alle Ausführungen sind auf das Wesentliche reduziert. ${ }^{5}$

\section{Das rätselhafte Genus der Lehrbekenntnisse - Bekenntnisse im Vorfeld der Confessio Augustana}

Bekanntlich ist das Entstehen von Lehrbekenntnissen ein ganz eigentümlicher Vorgang, der sich in den Reformationskirchen am Ende der 20er Jahre vollzieht und der zu einer Reihe von derartigen Texten führt, die in Form und Funktion sehr unterschiedlich sind. Während die überlieferten altkirchlichen Symbole zugleich eine gottesdienstliche Funktion hatten, ist das neue Textgenus des Lehrbekenntnisses für den gottesdienstlichen Gebrauch denkbar ungeeignet. Dieses neue Genus leitet sich her aus dem Bekenntnis, das Luther an das Ende seiner Abendmahlsschrift von 1528 stellt. ${ }^{6}$ Von dort aus führt die Linie über die Schwa-

5 Für alle näheren Begründungen und Differenzierungen verweise ich auf: Notger Slenczka, Theologie der reformatorischen Bekenntnisschriften, Leipzig 2020, zum Aufbau der CA bes. $\S 3$. 6 Martin Luther, Vom Abendmahl Christi. Bekenntnis [1528], BoA III, hg.v. Otto Clemen, Berlin ${ }^{6} 1966,352-516$, hier $507 \mathrm{ff}$. 
bacher Artikel (SchwabA) und über die von diesen abhängigen Marburger Artikel (MarbA) zur CA. In das unmittelbare Vorfeld gehören auch die Torgauer Artikel (TorgA).

Ich werde im Laufe des Referats eine These zum Grund für die Entstehung dieses Textgenus aufstellen. Hier greife ich auf diese Genealogie der CA nur zurück, weil sich damit Einsichten in den Aufbau der Bekenntnisse und die Leitprinzipien dieses Aufbaus gewinnen lassen. Luthers Bekenntnis von 1528 gibt das Grundprinzip vor: die spezifischen Entscheidungen, an denen die Interessen der reformatorischen Theologen haften - die Frage nach der Heilsvermittlung und die Frage nach dem Sinn der Sakramente - werden in den Aufriss der gottesdienstlich verwendeten altkirchlichen Symbole eingefügt. ${ }^{7}$

Wichtig für das Verständnis des Aufbaus der CA sind insbesondere die Schwabacher und die Marburger Artikel, die in gleicher Weise wie das Bekenntnis Luthers die spezifisch reformatorischen ,Lehrstücke' in den Aufriss der altkirchlichen Bekenntnisse einfügen. ${ }^{8}$ Wenn man nun aber den Aufbau der Schwabacher mit den Marburger Artikeln vergleicht, dann fallen auf den ersten Blick Umstellungen auf. ${ }^{9}$ Die Schwabacher Artikel lassen in der Abfolge der Artikel einen Gedankengang erkennen, der von der christologisch fokussierten Trinitätslehre in Art. 1 über die Schilderung des Heilswerkes Christi und dessen Rezeption im Glauben (Art. 2-5) zum Ursprung des Glaubens in Wort und Sakrament (Art. 6 Satz 1 und 7) führt und schließlich im zweiten Teil des Art. 6 bei der ethischen Neuorientierung als Folge des Glaubens endet. Darauf folgen in den Art. 7-10 die Konkretionen zu den Ursprüngen des Glaubens (Art. 6): Wortverkündigung und Sakramente. Die Marburger Artikel hingegen stellen um: die ersten Artikel ent-

7 Kurz aufgewiesen anhand des Bekenntnisses von 1528 (Luther, Vom Abendmahl Christi. Bekenntnis, 352-516, hier $507 \mathrm{ff}$.): Luther folgt grundsätzlich dem Aufriss der altkirchlichen Bekenntnisse (Trinitätslehre: 508,11-17; Christologie: 508,18-509,4; Pneumatologie: 511,13-20). Selbstverständliches wie die Schöpfungslehre und die diesem zugeordnete Rede von Gott, dem Vater, übergeht Luther, fügt aber in den zweiten Artikel die Feststellung der menschlichen Sünde (509,4-10), der Erlösung (10-14), der Ablehnung des freien Willens (15-23) und aller menschlichen Werke $(24-510,16)$ und die ,Stände‘ als dem eigentlichen Ort der Bewährung des Glaubens (510,17-40; Bekräftigung des sola fide 510,40 - 511,11) ein; in den Dritten Artikel ordnet Luther die Sakramente als Medien der Heilsvermittlung ein $(511,28-512,13)$. Es folgt eine wieder auf die Sakramente hinzielende (512,33 - 36) Ekklesiologie (512,14-41), die dann in eine Begründung der Ablehnung von Sakramenten und Sakramentalien hinausläuft [Hochschätzung der Beichte (513,1-12) und Ablehnung des Ablasses und der damit verbundenen Vorstellungen (513,13-32); Ablehnung der Heiligenverehrung (513,33-37); letzte Ölung etc.pp.]. Dazu Slenczka, Theologie, $168-172$.

8 Zum Folgenden vgl. Slenczka, Theologie, 164-166.

9 Slenczka, Theologie, 172-174. 
sprechen ziemlich klar dem in den SchwabA vollzogenen Weg von der Trinitätslehre (Art. 1) über die Christologie (2-3) zum Glauben (4 und 5 bzw. 7) und zu den Ursprüngen des Glaubens: Wort und Taufe (6;8-9). Dann stellen aber die Verfasser die Ausführungen zum neuen Leben gegenüber den SchwabA um und dem Taufartikel nach (10, vgl. SchwabA 6); dann erst folgt in MarbA der Artikel zur Buße (11), so dass sich hier ein am Lebensvollzug orientiertes Schema ergibt: Glaube (7) - Wort und Taufe als Ursprung des Glaubens (8 - 9) - neues Leben (10) - Buße (11). Diese Umstellung ist offensichtlich inhaltlichen Prinzipien geschuldet: um systematischer Gliederungsprinzipien willen gerät die Orientierung am Ablauf des altkirchlichen Glaubensbekenntnisses aus dem Blick. Dafür spricht auch, dass im Unterschied zu den SchwabA ein eigener Artikel zur Kirche und zum Jüngsten Gericht in den Marburger Artikeln fehlt.

Die Details sind weniger wichtig als die These, die sich daraus ableiten lässt: die Orientierung der reformatorischen Bekenntnistexte an der Abfolge des altkirchlichen Bekenntnisses löst sich fortschreitend auf zugunsten von systematischen Gliederungsaspekten, die den spezifischen Anliegen der reformatorischen Theologie Rechnung tragen.

\section{Zur Confessio Augustana und ihrem Aufbau}

Es ist schwer, zur CA etwas Neues zu sagen - das Folgende ist aber tatsächlich neu und wurde so noch nicht gesehen. Ich habe wichtige rezente Vorschläge zum Verständnis des Aufbaus der CA anderweitig ${ }^{10}$ vorgestellt und diskutiert und kann mich hier auf die Grundzüge der eigenen These beschränken und für nähere Begründungen ebenfalls auf anderweitig Vorgetragenes verweisen. ${ }^{11}$

\subsection{Probleme des Aufbaus}

Ich setze die Unterscheidung der Lehrartikel (1-21) von den spänigen Artikeln (22-28) als bekannt voraus; das hat zur Folge, dass die Frage nach dem Aufbau der CA sich auf die Art. 1-21 beschränken muss und kann.

Die Confessio Augustana ist auf den ersten Blick ein schlechter Kandidat für die Annahme, dass hier ein mit Sorgfalt und Finesse gestalteter Text vorliegt. Zunächst legt sich der Verdacht nahe, dass es eigentlich keine Aufbauprinzipien

10 Slenczka, Theologie, 182-190.

11 Slenczka, Theologie, 182-226. 
gibt; jedenfalls leitet nicht mehr der Aufriss der altkirchlichen Bekenntnisse: Der Artikel zur Erbsünde hat sich verselbständigt und ist zwischen die Rezeption der Gottes- und Trinitätslehre (CA 1) und die Christologie (CA 3) gerückt - in den SchwabA und in den MarbA war die Sündenlehre in den Artikel von der Heilszueignung - in beiden Bekenntnissen jeweils Art. 4 - integriert. Auch der Kirchenartikel ist im Vergleich mit SchwabA (dort 12) vorgerückt (CA 7) und leitet die Sakramentsartikel ein - die Schwabacher Artikel hatten die Artikel zum Glauben und zum Ursprung des Glaubens (SchwabA 5-11) als Einheit auf die Christologie (SchwabA 1-3) und deren soteriologische Pointe (4) folgen lassen und waren dann nach den Artikeln zu den Sakramenten (8-11) mit der Ekklesiologie (12) wieder zum Aufbau des altkirchlichen Bekenntnisses zurückgekehrt.

Die Tendenz zur Ablösung von der Themenfolge der altkirchlichen Bekenntnisse setzt sich also fort; zweitens ist auffällig, dass Wiederholungen auftreten: Auf CA 6 (,Vom neuen Gehorsam') folgt in CA 20 noch eine ausführliche Behandlung des Themas ,Vom Glauben und guten Werken'. In CA 18 wird ,Vom freien Willen' gehandelt, in CA 19,Von Ursach der Sünden` - obwohl diese Inhalte auch zwanglos in CA 2 unter dem Titel ,Von der Erbsünde“ hätten integriert oder angeschlossen werden können. Die Doppelungen setzen ein mit CA 14 (,Vom Kirchenregiment'): der Artikel führt inhaltlich Art. 5 (,Vom Predigtamt') weiter. Vermutlich darum verzichten manche Interpreten der CA - ich nenne nur Wilhelm Maurer und Leif Grane - völlig auf eine Analyse des Aufbaus.

\subsection{Vorschläge}

Die Vorschläge, die gemacht wurden, sind auch wenig befriedigend; ein Beispiel: Nach Bornkamm haben die ersten drei Artikel die Aufgabe, die Übereinstimmung der reformatorischen Kirchentümer mit den Lehrbildungen der Alten Kirche auszuweisen; daraufhin komme in den Artikeln 4-6 sowie 18-20 das reformatorische Verständnis des Evangeliums zur Darstellung. In 7-15 werden nach Bornkamm Probleme der Kirche und der kirchlichen Ordnung entfaltet. Hinzu kommen, so Bornkamm, drei „Einzelartikel, die dringende Fragen der Zeit klären sollen“ (11): Zum weltlichen Regiment in Art. 16, zur Wiederkunft Christi in 17 und zur Heiligenverehrung in $21 .{ }^{12}$

Das ist mehr Chaos als Aufbau; der Vorschlag Friedrich Winters, dass sich Melanchthon für den Aufbau der Artikel an der Abfolge der Teile des Sentenzenwerks des Petrus Lombardus orientiere, wird von einigen jüngeren Interpreten

12 Im RGG-Artikel spricht Bornkamm von „Einzelprobleme(n)“. 
aufgegriffen; der Vorschlag hat aber wirklich überhaupt gar nichts für sich und wird hoffentlich irgendwann vergessen. Zuweilen wird vorgeschlagen, dass Melanchthon in einem ersten Teil der Artikel die Übereinstimmung mit dem Glauben der Alten Kirche ausweise und ab Art. 4 oder ab Art. 5 die reformatorischen Sonderlehren begründe. ${ }^{13}$

Der etwas rätselhafte Aufbau erschließt sich aber, wenn man dem Hinweis Georg Kretschmars auf die Funktion und Stellung des Kirchenartikels (CA 7) folgt und die damit verbundene These etwas ausbaut. ${ }^{14}$

\subsection{Die Artikel 14-21}

Alle Interpreten haben jedenfalls Schwierigkeiten mit der Zuordnung der Art. 14-21 - das ist der Komplex, der deutliche thematische Wiederholungen bietet. Die einfachste Erklärung für diese Artikel ist die, dass Melanchthon hier Abgrenzungen gegen die 404 Artikel Johannes Ecks vornimmt. Eck hatte im Vorfeld des Reichstags eigene und fremde Zitat- und Thesensammlungen aus Schriften Luthers kompiliert und an den kaiserlichen Hof nach Innsbruck geschickt. Damit wurde die reformatorische Bewegung aller möglichen häretischen und politisch aufrührerischen Ansichten beschuldigt, unter anderem der Ablehnung einer ethischen Verpflichtung des Christen, einer Ablehnung der Obrigkeit und des Verteidigungskrieges gegen das Osmanische Reich. Für alle Abgrenzungen, die in den Art. 14-21 vorgenommen werden, lassen sich entsprechende Aussagen in den 404 Artikeln identifizieren. ${ }^{15}$ Die CA besteht also strenggenommen nicht aus zwei, sondern aus drei Teilen: den Lehrartikeln 1-21, innerhalb derer die Art. 1-13 von der Auseinandersetzung mit dem 404 Artikeln Ecks in 14-21 zu unterscheiden ist; und den ,spänigen' Artikeln 22-28. Das erklärt die von Art. 14 an auftretenden thematischen Doppelungen, und es führt dazu, dass man für die Frage nach dem Aufbau der CA sich an die Artikel 1-13 zu halten hat. $^{16}$

$13 \mathrm{Zu}$ Bornkamm: Slenczka, Theologie, 184f.; zu Winter: ebd. 185f.; zum zuletzt genannten Vorschlag: etwa Bornkamm (ebd. 184).

14 Ich nenne immer wieder gern den Aufsatz, in dem Kretschmar diese kluge Einsicht vorstellt (Slenczka, Theologie, 191): Georg Kretschmar, Der Kirchenartikel der Confessio Augustana Melanchthons, in: Erwin Iserloh (Hg.), Confessio Augustana und Confutatio (etc.), Münster 1980, 411-439, hier $417 \mathrm{f}$.

15 Dazu Slenczka, Theologie, 193-195.

16 Zum Folgenden: Slenczka, Theologie, 196-212. 


\subsection{Die Sakramentsartikel 8-13}

In diesem ersten Teil der CA hebt sich auf den ersten Blick ein Block von Artikeln ab, der dem Thema des Sakraments gewidmet ist. Dieser Block endet mit Art. 13, wo es um den Gebrauch der Sakramente geht - sie sind promissio und zielen auf den Glauben. Dem gehen die Artikel zum Bußsakrament und zur Bußgesinnung voraus, und natürlich Art. 9 und 10 zu Taufe und Eucharistie. Zu den Sakramentsartikeln gehört aber auch Art. 8, der aufgrund der sekundären und irreführenden Überschrift - „Was die Kirche sei?“ - üblicherweise gemeinsam mit Art. 7 dem Thema der Kirche zugeordnet wird. Das ist mit Rücksicht auf die Einleitung des Artikels auch zutreffend, aber der Artikel insgesamt hat, wie spätestens die Verwerfung zeigt, eine antidonatistische Spitze: er stellt die Gültigkeit von Sakramenten sicher, die von unwürdigen Priestern gereicht werden. Es geht also nicht um die Kirche, sondern um die Sakramente, so dass man es in den Art. 8-13 mit einem thematischen Bogen von der Spendung bis zum Empfang der Sakramente zu tun hat.

\subsection{Artikel 1-6}

\subsubsection{Artikel 4-6}

Es ist sodann deutlich, dass die Art. 5 und 6 nicht, wie vielfach vorgeschlagen wurde, einen Neueinsatz darstellen für einen Abschnitt, der von Art. 5 bis 17 reicht und der den Glauben, der in Art. 4 genannt wird, inhaltlich entfaltet. Sowohl Art. 5 wie Art. 6 verweisen durch eine Einleitung (,Solchen Glauben zu erlangen“ und „Auch wird gelehrt, dass solcher Glaube“) zurück auf Art. 4. Inhaltlich geht es in Art. 5 darum, dass der rechtfertigende Glaube eine Gabe Gottes ist, die durch Wort und Sakrament gegeben wird und keine verdienstliche Vorbereitung des Menschen erfordert; und es geht in Art. 6 darum, dass der Glaube ethische Folgen hat und haben muss, die aber auf keinen Fall als Grund der Rechtfertigung betrachtet werden dürfen. Es geht also in beiden Artikeln darum, den Ursprung und die Folgen des Glaubens unter das Vorzeichen der in Art. 4 skizzierten Rechtfertigung ohne das Werk zu stellen. Art. 4-6 gehören also engstens zusammen, mit Art. 5 fängt kein neues Thema an. ${ }^{17}$

17 Gegen den Vorschlag von Wenz, Slenczka, Theologie. Für diese Zuordnung der Art. 4 und 5 spricht übrigens auch die Vorform der Schwabacher Artikel: in SchwabA 6 werden die in CA 5 und 6 getrennten Anliegen verbunden; Slenczka, Theologie, $197 \mathrm{f}$. 


\subsubsection{Zentralstellung von Artikel 3 im Gefüge von 1-6}

Gemeinhin wird nun Art. 4 als das Zentrum der ersten Artikel der CA ausgegeben ich halte das für unzutreffend: das Zentrum ist vielmehr Art. 3. Es handelt sich um einen ganz wunderbar aufgebauten Artikel, der in genialer Weise die Art. 2-4 der Schwabacher Artikel ineinanderschiebt und Christologie und Soteriologie zu einer Einheit verbindet - ich verweise auf das Aufbauschema am Ende des Beitrags. ${ }^{18}$ Anderweitig habe ich in einer Detailanalyse begründet, dass die vier Artikel CA 1-4 untereinander eine Einheit bilden, in der nicht Art. 4, sondern Art. 3 das Zentrum darstellt; ich plausibilisiere das in abgekürzter Form durch das Identifizieren von Querverweisen: Der Artikel 3 weist im ersten Satz zurück auf Art. 1, in der Darstellung des Todes Jesu als Versöhnung des Gotteszorns auf Art. 2, und in der Doppelung, nach der der Tod Jesu als Grund für die Erlösung von der Sünde und das Auferstehungsleben Jesu als Grund des neuen Lebens des Christen beschrieben wird, auf die entsprechende Doppelung in Art. 4: dort wird dem entsprechend festgestellt, dass die Vergebung der Sünde einerseits und Gerechtigkeit und Ewiges Leben andererseits allein dem Glauben entspringe. Eine detaillierte Analyse lässt sich in der Feststellung zusammenfassen, dass die Artikel 1-4 eine Einheit bilden, die von der Christologie regiert wird. Es geht hier um den $\mathrm{Zu}$ sammenhang des Evangeliums von Christus mit dem Glauben. Art. 5 und 6 sind, wie gesagt, von Art. 4 abhängig.

\subsection{Zentralstellung von Artikel 7 im Rahmen von 1-13}

Damit haben wir zwei Blöcke von je sechs Artikeln, die thematisch zusammenhängen, Art. 1- 6 unter dem Thema von Christus und Glaube, Art. 8-13 unter dem Thema des auf Glauben zielenden Sakraments. Evangelium von Christus einerseits. Sakrament andererseits. Beides jeweils fokussiert auf den Glauben, auf den sie abzielen. Und in der Mitte der Kirchenartikel, in dem festgestellt wird, dass die Kirche die Gemeinschaft der Glaubenden ist, die dort ist, wo das Wort - das ist das Evangelium von Christus - recht verkündigt und die Sakramente dem Evangelium gemäß verwaltet werden. Also: in der Mitte steht der Kirchenartikel, der die Kirche als Gemeinschaft der Glaubenden definiert. Drumherum die Konstitutionsgründe der Gemeinschaft der Glaubenden: Wort von Christus. Und das Sakrament. Und genau dies ist der Grund dafür, dass Melanchthon den Kirchenartikel von dem Ort, an dem er nach dem altkirchlichen Bekenntnis und nach den diesem fol-

18 Zur Entfaltung dieses Schemas: Slenczka, Theologie, 202-204. 
genden Schwabacher Artikeln steht, wegrückt und vor die Sakramentenartikel stellt. Die Orientierung des Aufbaus am altkirchlichen Glaubensbekenntnis ist gänzlich einer systematisch begründeten Abfolge gewichen: im Zentrum die Kirche, gerahmt von deren Konstitutionsgründen.

\section{Das systematische Zentrum der Confessio Augustana: die Definition der Kirche}

Damit ist das Zentrum der CA der Kirchenartikel. Ich sortiere ihn noch kurz ein. ${ }^{19}$

\subsection{Die Kirche als ,Versammlung der Gläubigen'. Wort und Sakrament als Erkennungszeichen der Kirche}

Im Zentrum des Kirchenartikels steht eine Definition der Kirche, nämlich als ,Versammlung aller Gläubigen'. Damit wird bekanntlich die entsprechende Formulierung des Apostolikums aufgenommen - communio sanctorum. Diese Wendung ist doppeldeutig und wird von der Mehrheit der vorreformatorischen Theologen als ,Gemeinschaft heiliger Dinge، im Sinne der Teilhabe an den Sakramenten gedeutet. Die lateinische Fassung klärt die Doppeldeutigkeit in der Richtung eines Verständnisses der Kirche als Personengruppe, indem Melanchthon nicht communio, sondern congregatio sanctorum schreibt. Die ursprüngliche deutsche Fassung wiederum definiert die Heiligkeit durch den Glauben: die congregatio sanctorum ist die „Versammlung aller Glaubigen“; entsprechend wird Luther später in Von den Konziliis und Kirchen im dritten Teil die Heiligkeit der Kirche durch den Glauben definieren, weil in diesem, so legt er in einem absolut genialen, weil wunderbar aufgebauten Abschnitt aus, alle Gebote erfüllt seien. ${ }^{20}$ Ich verweise auch darum auf diesen Text, weil Luther dort feststellt, dass diese Versammlung der Gläubigen unsichtbar sei, da der Glaube nicht augenfällig sei, so dass sich die Frage stelle, wo eigentlich diese Gemeinschaft der Gläubigen sei; und er nennt dort Erkennungszeichen der Kirche, voran Wort und Sakrament, mit der Begründung, dass durch diese eben der Glaube entstehe, der das Wesens-

19 Dazu Slenczka, Theologie, 207-212.

20 Martin Luther, Von den Conciliis und Kirchen (1539), DDStA 2, hg.v. Dietrich Korsch/Johannes Schilling, Leipzig 2015, 527-799, hier Teil 3 (742ff.), zur Kirchendefinition und zum Verhältnis von Heiligkeit und Glaube: 742,26-744,4. Zur ,Heiligkeit‘ als Implikat des Glaubens (dies ist der wunderbar aufgebaute Abschnitt): 746,21-748,22. 
element der Kirche sei. ${ }^{21}$ Aus demselben Grund werden hier in der CA Wort und Sakrament genannt: sie sind Erkennungszeichen der Kirche, und zwar deshalb, weil durch sie Glaube entsteht. Dass irgendwo mit hoher Wahrscheinlichkeit Glaube und damit die Gemeinschaft der Glaubenden ist, erkennt man daran, dass dort die Medien sind, durch die dieser Glaube entsteht.

\subsection{Der Anspruch der reformatorischen Kirchentümer auf den Titel ,Kirche}

Damit erschließt sich der Sinn der CA: dadurch, dass die weltlichen Herren den Anspruch erheben, dass in ihren Kirchen ein magnus consensus besteht über die Artikel, in denen die angemessene Evangeliumsverkündigung und die Grundlagen einer dem Evangelium entsprechenden Sakramentsverwaltung beschrieben werden, erheben sie gleichzeitig den Anspruch, dass in ihren Kirchentümern die heilige christliche Kirche sei auch dann, wenn sie hinsichtlich der von Menschen eingesetzten Zeremonien von der römischen Kirche abweicht.

\subsection{Kirche ohne konstitutives Bischofsamt}

Und damit ist auf der anderen Seite deutlich: wir haben es hier in CA 7 mit einer Kirchendefinition zu tun, die den Anspruch erhebt, hinreichend zu sein - satis est. Sie ist somit nicht vollständig, zweifellos. Zur Kirche gehört mehr als die Verkündigung des Evangeliums und die entsprechende Sakramentsverwaltung. Zur Kirche gehört ein Versammlungsgebäude, zweifellos auch Menschen, die für die Verkündigung und die Sakramentsverwaltung zuständig sind, Vereinbarungen über ein regelmäßiges Angebot dieser Medien etc.pp. Einiges davon wird auch in den anderen Artikeln genannt - so etwa das kirchliche Amt in Art. 5, aber eben: der Entstehung des Glaubens durch Verkündigung und der Sakramentsverwaltung nachgeordnet: es ist das ministerium docendi et porrigendi sacramenta, wie es im lateinischen Text heißt. ${ }^{22}$ Genau so wird durchgängig die Amtsstruktur der

21 Luther, Von den Conciliis, 750,27-752,2; es folgen dann die Erkennungszeichen: Wort (752,3754,29); Taufe (754,30-756,12); Eucharistie (756,13-758,5); Buße (758,6-760,5); Berufung von Amtsträgern (760,6-776,6); öffentlicher Gottesdienst (776,7-23); Martyrium (776,24-778,31). Zusammenfassung: 778,32-780,39.

22 Man müsste ,Predigtamt‘ als ,Aufgabe der Predigt‘ übersetzen; ich verweise auf die entsprechende Passage aus den SchwabA, in der das ,Predigtamt‘, das Gott eingesetzt habe, mit einem explikativen ,oder verbunden wird mit dem ,muntlich wort': „Sollichen glauben zu erlangen oder 
Kirche behandelt: das Amt ist nicht die Kirche, so wenig, wie die Verkündigung und die Sakramente die Kirche sind, sondern die Kirche ist die Gemeinschaft der Glaubenden, konstitutiv ist der Glaube. Wort und Sakrament sind die unverzichtbaren Medien durch die der Glaube entsteht, und das Amt ist dem zugeordnet als Amt der Verkündigung und Sakramentsverwaltung - das sind in nuce die Ausführungen zum Bischofsamt in Art. 28.

Um es ganz klar zu sagen: wir haben in Art. 7 eine Definition der Kirche vor uns, die hinreichend sein will, dabei aber ohne jeden Bezug zum bischöflichen Amt auskommt. Es wird nicht einmal erwähnt, und daran ändert auch Art. 5 und 8 nichts; in beiden geht es nicht darum, das bischöfliche Amt als konstitutives Moment in die Definition der Kirche einzuzeichnen. Dass die Evangeliumsverkündigung und die Sakramentsverwaltung irgendwie gewährleistet sein muss und dafür Personen zuständig sein müssen, ist klar. Aus der Definition der Kirche als Gemeinschaft der Glaubenden leiten die Verfasser der CA die Konstitutionsgründe dieses Glaubens als Erkennungszeichen ab. Eine Wahrnehmung dieser Erkennungszeichen durch Amtsträger ist impliziert. Eine bestimmte Ausstattung des Amtes gehört aber nicht zu den konstitutiven Wesensmerkmalen der Kirche, sondern zu den Adiaphora. ${ }^{23}$

\section{Gegenwärtige Relevanz: ,Kirche`als Begriff und als Name}

Damit komme ich zum dritten im Titel meines Vortrags genannten Aspekt, nämlich zur Gegenwartsrelevanz der CA.

\subsection{Die Kirche als civitas platonica - das Recht eines Einwandes des Kardinal Bellarmin}

Ich habe das ökumenische Programm der Tagung durchaus wahrgenommen, möchte aber nun doch etwas Wasser in den Wein schütten, indem ich mich auf

uns menshn zugeben, hat Got eingesetzt das predig ambt oder muntlich wort, nemlich das evangelion [...]“: es geht vor diesem textgeschichtlichen Hintergrund auch in CA 5 also nicht um die Einsetzung eines Amtsstandes, sondern um die Einrichtung einer Aufgabe: der Verkündigung des Evangeliums. Das Geistliche Amt wird nicht in CA 5 begründet, sondern in CA 14 - so zu Recht DorotheaWendebourg, Das Amt und die Ämter, in: ZevKR 45 (2000), 5-38.

23 Dazu genauer und im Abgleich mit den übrigen Aussagen der CA und der auf sie folgenden Bekenntnisschriften zum geistlichen Amt: Slenczka, Theologie, 213-223. 
den großen und ganz ernsthaft nicht genug zu lobenden Kardinal Bellarmin beziehe. ${ }^{24}$ Er hat das Kirchenkonzept der Protestanten als das Modell einer civitas platonica abgelehnt - dieser Vorwurf wird, soweit ich sehe, in den Auseinandersetzungen der frühen Reformationszeit erstmals erhoben; der erste mir bekannte Beleg stammt von Johannes Eck. Bellarmin hat, wie mit so vielem, recht mit dieser Charakterisierung der reformatorischen Ekklesiologie, wenn man diese Bezeichnung richtig versteht. Bellarmin hebt damit nämlich darauf ab, dass die Protestanten durchschnittlicherweise die Kirche als Allgemeinbegriff verstehen. Allgemeinbegriffe zeichnen sich dadurch aus, dass sie auf viele Entitäten anwendbar sind, sofern diese die im Begriff angelegten Bedingungen erfüllen. Alle, die diesen Text lesen oder lesen werden, sind Menschen, sofern sie die Bedingung erfüllen, dass sie vernünftig sind, und dass sie sich von anderen Vernunftwesen den Engeln, den Teufeln, oder auch Gott - dadurch unterscheiden, dass sie einen Körper haben, sich bewegen, einen Platz im Raum einnehmen und Stoffwechselvorgänge aufrechterhalten, kurz: dass sie Lebewesen sind. Sofern sie das sind und tun, sind sie Menschen, unbeschadet aller Differenzen bezüglich der Hautund Haarfarbe, Nasenform, Körperumfang oder Geschlechtszugehörigkeit. Es ist genug - satis est! - wenn sie vernünftig und ein Lebewesen sind. In diesem Sinne ist nach protestantischem Verständnis die Kirche eine civitas platonica: das Kirchesein einer Gemeinschaft entscheidet sich daran, dass diese Gemeinschaft bestimmte, im Begriff angelegte Bedingungen erfüllt, nämlich dass es sich um eine Gemeinschaft von Glaubenden handelt - und diese erkennt man eben daran, dass in ihr Wort und Sakrament gepflegt wird.

Bellarmin erhebt den Anspruch, dass er und die römische Kirche insgesamt die Kirche nicht als civitas platonica versteht, das heißt: nicht als Begriff verwendet. Nun sieht es aber auf den ersten Blick doch so aus, als ob die römischen Katholiken ebenfalls einen solchen Allgemeinbegriff von Kirche hätten: Dass sie ein Set von Merkmalen und Kriterien mit den protestantischen Kirchen teilten, dabei aber über diese unstrittigen Merkmale der Kirche bzw. Kriterien des Kircheseins hinaus weitere Kriterien für das Kirchesein hinzufügten. Bellarmin nennt schließlich auch solche Wesenselemente der Kirche, nämlich das gemeinsame Bekenntnis des Glaubens, die Gemeinschaft der Sakramente, und den Gehorsam gegenüber den legitimen Bischöfen, zuhöchst gegenüber dem Papst zu Rom. Eindeutig im Zentrum steht dabei das Amt in apostolischer Sukzession - das sind

24 Die folgenden Überlegungen zu Bellarmin und zur Differenz des Verständnisses eines Wortes als Begriff und als Name habe ich ausführlicher entfaltet: Notger Slenczka, Die trennende Kraft der einen Kirche. Probleme der ekklesiologischen Grundlagen der Ökumene, in: Reinhard Flogaus u.a. (Hg.), Orthodoxie im Dialog. Historische und aktuelle Perspektiven. FS für Heinz Ohme, Leipzig 2015, 121-147. 
die legitimi pastores, denn dieses Kriterium ist das im Vergleich mit den anderen beiden engste. Man denke hinsichtlich der Bedeutung dieses Kriteriums an die Debatten um die Kriterien des Kircheseins im Anschluss an die Gemeinsame Erklärung zur Rechtfertigung: nicht allein das Verständnis der Rechtfertigung, sondern auch andere Kriterien (genannt wurde ausdrücklich das apostolische Amt) sind konstitutiv für die Zugehörigkeit zur Kirche. Versteht man das Bekenntnis, die Teilnahme am Sakrament und den Gehorsam gegenüber dem Amt als konstitutive Momente der Kirche, dann wäre auch der Kirchenbegriff der römischen Kirche ein Allgemeinbegriff, der aber im Vergleich mit den protestantischen Kriterien weitere nennt mit der Folge, dass möglicherweise eine kleinere Zahl von Gemeinschaften wirklich als Kirche bezeichnet werden kann: nur diejenigen, die neben Wort und Sakrament auch noch die apostolische Sukzession aufweisen. Wenn die Sachlage wirklich so stünde, dann wäre ein ökumenischer Dialog über den genauen Sinn des Begriffes Kirche notwendig und möglicherweise erfolgreich: man könnte dann fragen, ob wirklich die Amtssukzession zum Begriff der Kirche gehört, und ob nicht das Amt irgendwo doch auch in der CA als Bedingung des Kircheseins unterzubringen ist.

\subsection{Kirche als ,Name}

Aber wir haben es eben nicht damit zu tun, dass alle Denominationen den Begriff Kirche in gleicher Weise verwenden und nur unterschiedlich definieren und damit den Begriff unterschiedlich weit anwenden. Sondern wir haben es mit einer schon rein formalsprachlich unterschiedlichen Verwendung des Kirchenbegriffes zu tun. Denn gerade dadurch, dass die römisch-katholische Kirche die apostolische Sukzession zu den konstitutiven Eigenschaften der Kirche rechnet, verwendet sie den Begriff Kirche nicht nur als Allgemeinbegriff, sondern grundlegend als Namen, genaugenommen als Eigennamen. Eigennamen zeichnen sich dadurch aus, dass sie von genau einer Entität gelten, sie deuten genau auf eine bestehende Entität, und dieses Deuten auf eine bestimmte Entität ist konstitutiv für ihren Sinn. Wer Notger Slenczka sagt, der definiert damit nicht Kriterien (Haarfarbe, Größe, Leibesumfang) und sagt: alle, die diesen Kriterien genügen, sind Notger Slenczka, sondern Notger Slenczka ist ein deiktischer Begriff wie ,dies“oder ,hier und ,jetzt‘: man zeigt mit dem Begriff auf ein Einzelexemplar. Auch wenn es mehrere Einzelexemplare dieses Namens gäbe, würde dadurch aus dem Namen kein Allgemeinbegriff, denn es verbindet sich mit dem Gebrauch des Namens nicht der Anspruch, dass diese Einzelexemplare eine ,Notger-Slenczka-haftigkeit“ gemeinsam hätten, die sie von der ,Friedrich-Nietzsche-haftigkeit‘ einer anderen Individuengruppe unterscheidet. Ein Eigenname bezeichnet nur mich. 
Die römisch-katholische Kirche - und übrigens auch die orthodoxen Kirchen - bezeichnen mit dem Begriff Kirche eine bestehende Wirklichkeit und nur diese, nämlich exakt die von Christus ausgehende und berufene Gemeinschaft, deren Kontinuität und Identität im Zeitverlauf durch die (beanspruchte) lückenlose Folge und die wechselseitige Gemeinschaft der Nachfolger der Apostel gewährleistet ist und die in Gestalt der römisch-katholischen Kirche bzw. der orthodoxen Kirchen in der Gegenwart vor uns steht. „Kirche“ ist der Name für diese feststellbare Gemeinschaft, kein Allgemeinbegriff, der noch auf weitere Gemeinschaften Anwendung finden könnte. Und daher ist es mitnichten anstößig, sondern völlig folgerichtig und zutreffend, dass in diesem Sinne die protestantischen Kirchen nicht Teil der Kirche sind. Das sind wir nach römisch-katholischem Verständnis nicht, und das sind wir nach unserem eigenen auch nicht. Wir sind zwar Kirche, aber eben nicht in diesem Sinne, und die geradezu beleidigten Reaktionen der protestantischen Kirchenfürsten auf die Erklärung Dominus Jesus der Glaubenskongregation hat zu Recht Kopfschütteln auf sich gezogen.

Diese Verwendung des Begriffs „Kirche“ als Eigenname schließt nun natürlich nicht aus, sondern ein, dass dieser Begriff weitere semantische Gehalte in sich schließt - die Einheit, Heiligkeit, und Katholizität ebenso wie das unfehlbare Sein in der Wahrheit; diese Gehalte kommen nun aber eben als exklusive Eigenschaften und Prärogative dieser bestimmten Gemeinschaft zu stehen; und diese Bezugnahme auf eine bestimmte Gemeinschaft schließt zweitens auch nicht aus, dass etwa von der römischen Kirche anerkannt wird, dass es Momente der Wahrheit auch in anderen Gemeinschaften finden, die aber nicht schon allein dadurch Teil der Gemeinschaft sind und sein können, die der Name „Kirche“ bezeichnet.

\section{Das Kirchenverständnis der Confessio Augustana und der Grund für das Genus des Lehrbekenntnisses}

Zurück zur CA: Die CA verwendet in ihrem Zentrum, Art. 7, das Wort „Kirche‘ als Allgemeinbegriff, explizit in der aus den über die Torgauer Artikel von den Schwabacher Artikeln ererbten Feststellung, dass die Kirche immer und allenthalben (perpetua mansura) sei, was impliziert, dass man sie eben hier und da entdecken kann, wenn man auf die Erkennungszeichen achtet. Natürlich gilt das nach römischem Verständnis auch - aber eben in dem Sinne, dass es zu jedem Zeitpunkt die ungebrochene Kontinuität des Amtes gibt, an der alle sonstigen Kirchenepitheta hängen. Ich sage das übrigens ganz ohne negativen Unterton - 
das ist ein schlüssiges und durchdachtes Kirchenkonzept auch dann, wenn es nicht meines oder das der protestantischen Kirchen ist.

Die reformatorischen Kirchen hingegen konnten sich nicht mehr als Teil der einen Kirche im Sinne des durch den Eigennamen bezeichneten Einzelexemplars verstehen, denn ihnen fehlte das bischöfliche Amt, das die Kontinuität zur einen Kirche herstellte. Genau darum gibt es - das sei der abschließende Gedanke ${ }^{25}$ - in den protestantischen Kirchen das Institut der Lehrbekenntnisse: sie sind Kontinuitätskriterien, erheben den Anspruch, die Kontinuität zu den Aposteln nicht im Medium der Amtssukzession, sondern im Medium des Verständnisses des Evangeliums und der Sakramente herzustellen, kurz: im Medium der Lehre. Und genau dies ist der Grund dafür, dass der Term ,Kirche“ gleichsam die Wortart wechselt: vom Namen zum Begriff wird.

\section{Ist die Differenz im Kirchenverständnis überbrückbar?}

Ich halte diese Differenz im Verständnis der Kirche für tiefgreifend und für nicht aufhebbar: auch im wünschenswert freundlichsten ökumenischen Dialog ist es nicht vermittelbar, wenn ein Wort kategorial anders - als Begriff oder als Name verwendet wird. Vielmehr werden wir entweder dem jeweils anderen Verständnis des Wortes ,Kirche‘ beitreten, oder uns mit dieser Differenz abfinden müssen. Das ist aus protestantischer Perspektive nicht weiter aufregend oder bedauerlich. Denn natürlich sind wir einerseits im Sinne des skizzierten katholischen Kirchenbegriffs nicht Kirche oder Teil der Kirche, und wir wollen das auch gar nicht sein: es ist die Pointe der Kirchendefinition nach CA 7, dass das Kirchesein nicht an der Zugehörigkeit zu jenem durch die apostolische Sukzession zur Einheit werdenden ,Baum‘ hängt. Natürlich irrt nach unserem Selbstverständnis die katholische Kirche mit der These, dass wir nicht Kirche sind; sie kann aber eben unter ihren Voraussetzungen nicht anders, als solchermaßen zu irren. Umgekehrt ist die römisch-katholische Kirche nach unseren Kriterien selbstverständlich Kirche, wenn und wo Wort und Sakrament in ihr sachgemäß ausgerichtet werden. Und ich persönlich würde das so formulieren: die römisch-katholische Kirche ist selbstverständlich Kirche, weiß aber eben in ihrem offiziellen Selbstverständnis nicht, warum.

Eine Anerkennung der CA durch die katholische Kirche aber wäre nur unter Verzicht auf den römisch-katholischen Kirchenbegriff möglich. Wer eine solche

25 Vgl. Slenczka, Theologie, 124-126 und 698f. 
Anerkennung wünscht, wünscht den Übertritt der katholischen Kirche zur Reformation. Das wäre eine charmante Idee und durchaus zu begrüßen, wäre aber als eine im ökumenischen Gespräch geäußerte Erwartung doch eine eher unanständige Zumutung... 


\section{Anhang: Aufbau von Confessio Augustana 3 (Slenczka, Theologie, 203)}

Item, es wird gelehrt

[1. InKARNATION]

[PERSON CHRISTI]

daß Gott der Sohn sei Mensch worden, geborn aus der reinen Jungfrauen Maria

[2. Zwei-Naturen-Lehre]

und daß die zwo Natur, die gottlich und menschlich, in einer Person also unzertrennlich vereiniget,

ein Christus seind, welcher wahr Gott und wahr Mensch ist,

\section{[WERK CHRISTI (a) und das HEIL DES MENSCHEN (b)]}

[3. (a) Status Exinanitionis]

wahrhaftig geboren, gelitten, gekreuzigt, gestorben und begraben,

[3. (b) Heilsbedeutung des Status]

daß er ein Opfer wäre nicht allein fur die Erbsund, sunder auch fur alle andere Sunde und Gottes Zorn versohnet;

\section{[4. (a) Status Exaltationis]}

item, daß derselbig Christus sei abgestiegen zur Helle, wahrhaftig am dritten Tag von den Toten auferstanden, aufgefahren gein Himmel, sitzend zur Rechten Gottes,

[4. (b) Heilsbedeutung des Status]

1. daß er ewig herrsche uber alle Kreaturen und regiere,

2. daß er alle, so an ihne glauben, durch den heiligen Geist heilige, reinige stärke und troste, ihnen auch Leben und allerlei Gaben und Guter austeile und wider den Teufel und wider die Sunde schutze und beschirme;

[5. (a) Wiederkunft]

item, daß derselbig Herr Christus endlich wird offentlich kommen,

[5. (b) Heilsbedeutung der Wiederkunft]

zu richten die Lebendigen und die Toten etc., lauts des Symboli Apostolorum. 